\title{
Heat flow in the postquasistatic approximation
}

\author{
B. Rodríguez-Mueller, ${ }^{1}$ C. Peralta,${ }^{2,3}$ W. Barreto, ${ }^{4}$ and L. Rosales ${ }^{5}$ \\ ${ }^{1}$ Computational Science Research Center, College of Sciences, \\ San Diego State University, San Diego, California, USA \\ ${ }^{2}$ Deutscher Wetterdienst, Frankfurter Str. 135, 63067 Offenbach, Germany \\ ${ }^{3}$ School of Physics, University of Melbourne, Parkville, VIC 3010, Australia \\ ${ }^{4}$ Centro de Física Fundamental, Facultad de Ciencias, Universidad de Los Andes, Mérida, Venezuela* \\ ${ }^{5}$ Laboratorio de Física Computacional, Universidad Experimental \\ Politécnica "Antonio José de Sucre", Puerto Ordaz, Venezuela
}

(Dated: April 23, 2022)

\begin{abstract}
We apply the postquasistatic approximation to study the evolution of spherically symmetric fluid distributions undergoing dissipation in the form of radial heat flow. For a model which corresponds to an incompressible fluid departing from the static equilibrium, it is not possible to go far from the initial state after the emission of a small amount of energy. Initially collapsing distributions of matter are not permitted. Emission of energy can be considered as a mechanism to avoid the collapse. If the distribution collapses initially and emits one hundredth of the initial mass only the outermost layers evolve. For a model which corresponds to a highly compressed Fermi gas, only the outermost shell can evolve with a shorter hydrodynamic time scale.
\end{abstract}

PACS numbers: 04.25.-g,04.25.D-,0.40.-b

\section{INTRODUCTION}

Dissipation as an emission process [1] is crucial for the outcome of gravitational collapse. Thermal conduction is usually considered proportional to the gradient of temperature. This is a sensible choice, since the mean free path of particles responsible for the propagation of energy in stellar interiors is very small as compared with the typical length of the object [2]. Observations from supernova $1987 \mathrm{~A}$ indicate that the regime of radiation transport prevailing during the emission process is closer to the diffusion approximation than to the free streaming limit [3]. The addition of a test bed for studying dissipation mechanisms and other transport processes in order to later incorporate them into a more sophisticated numerical framework (Arnowitt-Deser-Misner [ADM] or characteristic) is a necessity.

In this work we study a self-gravitating spherical distribution of matter containing a dissipative fluid in the diffusion limit. We found behaviors similar to those reported with a different mechanism by [4, and report the zeroth order results for dissipation. We use noncomoving coordinates and follow the method reported in [5, 6] named the postquasistatic approximation (PQSA), which has been proposed as a test bed in numerical relativity [7. For recent advances and applications see [8] and 9]. For origin, reviews and details of the PQSA see [10, [11][15] and [16]-19]. We do not consider here temperature profiles to determine which processes can take place during the collapse. For that purpose, transport equations in the relaxation time approximation have been proposed to avoid pathological behaviors (see for instance [20] and

${ }^{*}$ On sabbatical leave. references therein). These issues will be considered in a future investigation. It is worth mentioning here that in order to get a higher order approximation we have to know the zero order approximation in the relaxation time, as in the present study. To the best of our knowledge, no author has undertaken in practice the dissipative matter problem in numerical relativity. Our purpose here is to show how heat flow processes can be considered in the context of the PQSA. The results indicate that an observer using radiation coordinates does not "see" some details when heat flow is considered. The final goal is to eventually study the same problem using the MüllerIsrael-Stewart theory for the dissipative system, which is highly nontrivial in spherical symmetry.

In Sec. II, we present the field equations, the matching conditions and the set of surface equations. For additional details concerning the PQSA method see [6] and 4. Three models are presented in Sec. III and some remarks are discussed in Sec. IV.

\section{MAIN EQUATIONS}

To write the Einstein field equations we use the line element in Schwarzschild-like coordinates

$$
d s^{2}=e^{\nu} d t^{2}-e^{\lambda} d r^{2}-r^{2}\left(d \theta^{2}+\sin ^{2} \theta d \phi^{2}\right)
$$

where $\nu=\nu(t, r)$ and $\lambda=\lambda(t, r)$, with $(t, r, \theta, \phi) \equiv$ $(0,1,2,3)$.

In order to get physical input we introduce the Minkowski coordinates $(\tau, x, y, z)$ by 21 .

$$
d \tau=e^{\nu / 2} d t, d x=e^{\lambda / 2} d r, d y=r d \theta, d z=r \sin \theta d \phi
$$

In these expressions $\nu$ and $\lambda$ are constants, because they have only local values. 
Following the Bondian point of view as in [21, 6], 7] and 4] we assume that, for an observer moving relative to the local Minkowskian coordinates with velocity $\omega$ in the radial direction, the space contains an isotropic fluid of energy density $\rho$, radial pressure $p$, and radial heat flux $q$. For this comoving observer, the covariant energy tensor in Minkowski coordinates is thus

$$
\left(\begin{array}{cccc}
\rho & -q & 0 & 0 \\
-q & p & 0 & 0 \\
0 & 0 & p & 0 \\
0 & 0 & 0 & p
\end{array}\right)
$$

Making a Lorentz boost we write the field equations in relativistic units $(G=c=1)$ as follows [4]:

$$
\begin{gathered}
\tilde{\rho}=\frac{1}{8 \pi r}\left[\frac{1}{r}-e^{-\lambda}\left(\frac{1}{r}-\lambda_{, r}\right)\right] \\
\tilde{p}=\frac{1}{8 \pi r}\left[e^{-\lambda}\left(\frac{1}{r}+\nu_{, r}\right)-\frac{1}{r}\right] \\
p=-\frac{1}{32 \pi}\left\{e^{-\lambda}\left[2 \nu_{, r r}+\nu_{, r}^{2}-\lambda_{, r} \nu_{, r}+\frac{2}{r}\left(\nu_{, r}-\lambda_{, r}\right)\right]\right. \\
\left.e^{-\nu}\left[2 \lambda_{, t t}+\lambda_{, t}\left(\lambda_{, t}-\nu_{, t}\right)\right]\right\} \\
S=-\frac{\lambda_{, t}}{8 \pi r} e^{-\frac{1}{2}(\nu+\lambda)},
\end{gathered}
$$

where the comma (,) represents partial differentiation with respect to the indicated coordinate and the conservative variables are

$$
\begin{gathered}
\tilde{\rho}=\frac{\rho+p \omega^{2}}{1-\omega^{2}}+\frac{2 q \omega}{1-\omega^{2}}, \\
S=(\rho+p) \frac{\omega}{1-\omega^{2}}+q \frac{1+\omega^{2}}{1-\omega^{2}}
\end{gathered}
$$

and the flux variable

$$
\tilde{p}=\frac{p+\rho \omega^{2}}{1-\omega^{2}}+\frac{2 q \omega}{1-\omega^{2}} .
$$

as in the standard ADM $3+1$ formulation. Within the PQSA $\tilde{\rho}$ and $\tilde{p}$ are referred as to effective density and effective pressure, respectively. Note that from (2) the velocity of matter in Schwarzschild coordinates is

$$
\frac{d r}{d t}=\omega e^{(\nu-\lambda) / 2}
$$

It is easy to check that [2]

$$
p_{a}=q_{a},
$$

which expresses the continuity of the radial pressure across the boundary of the distribution $r=a(t)$. Equivalently, in terms of the effective variables

$$
\tilde{p}_{a}=\tilde{\rho}_{a} \omega_{a}^{2}+q_{a}\left(1+\omega_{a}\right)^{2} .
$$

Defining the mass function as

$$
e^{-\lambda}=1-2 m / r
$$

and substituting (14) into (4) and (7) we obtain, after some rearrangements,

$$
\frac{d m}{d t}=-4 \pi r^{2}\left[\frac{d r}{d t} p+q(1-2 m / r)^{1 / 2} e^{\nu / 2}\right] .
$$

This equation is the momentum constraint in the ADM $3+1$ formulation, it expresses the power across any moving spherical shell.

Equation (6) can be written as $T_{1 ; \mu}^{\mu}=0$ or equivalently, after a lenghty calculation

$$
\begin{gathered}
\tilde{p}_{, r}+\frac{(\tilde{\rho}+\tilde{p})\left(4 \pi r^{3} \tilde{p}+m\right)}{r(r-2 m)}+\frac{2}{r}(\tilde{p}-p) \\
=\frac{e^{-\nu}}{4 \pi r(r-2 m)}\left(m_{, t t}+\frac{3 m_{, t}^{2}}{r-2 m}-\frac{m_{, t} \nu_{, t}}{2}\right) .
\end{gathered}
$$

This last equation is the generalization of the TolmanOppenheimer-Volkov for nonstatic radiative situations. In can be shown that Eq. 16 is equivalent to the equation of motion for the fluid in conservative form in the standard ADM 3+1 formulation [7].

At the surface, Eqs. (11), 15 and (16) lead us to a set of differential equations for $a, m_{a}$ and $\omega_{a}$ if we prescribe in some way the metric functions $(m$ and $\nu)$.

The other two field equations (4) and (5) can be integrated to obtain

$$
m=\int_{0}^{r} 4 \pi r^{2} \tilde{\rho} d r
$$

which is the Hamiltonian constraint in the ADM $3+1$ formulation and

$$
\nu=\nu_{a}+\int_{a}^{r} \frac{2\left(4 \pi r^{3} \tilde{p}+m\right)}{r(r-2 m)} d r,
$$

the polar slicing condition, from where it is obvious that for a given radial dependence of the effective variables, the radial dependence of the metric functions becomes completely determined.

\section{MODELING}

We consider here a seed model inspired by the wellknown Schwarzschild interior solution. This model corresponds to an incompressible fluid departing from the static equilibrium. Following the PQSA we take

$$
\tilde{\rho}=f(t)
$$




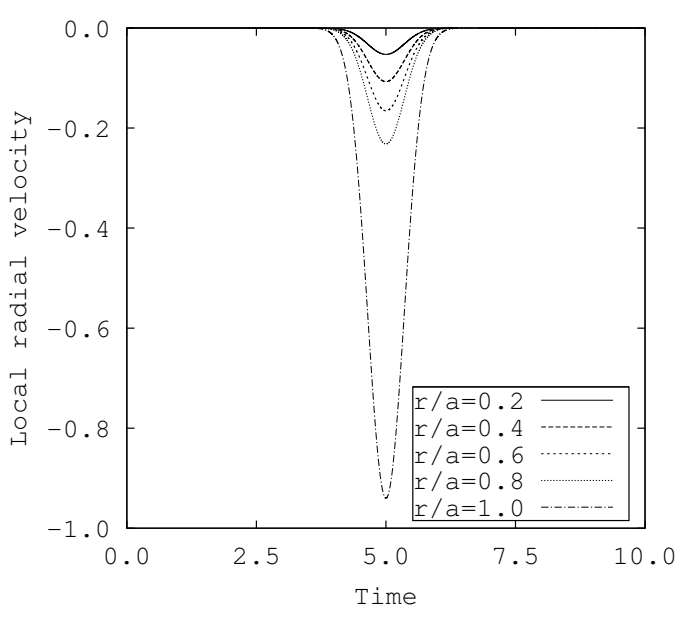

FIG. 1: Evolution of the radial local velocity $\omega$ (multiplied by $10^{3}$ ) for the Schwarzschild-like model. The initial conditions are $a(0)=5.0, m(0)=1.0, \omega_{a}(0)=0.0$. The total radiated mass is $M_{r}=10^{-4} m_{a}(0)$.

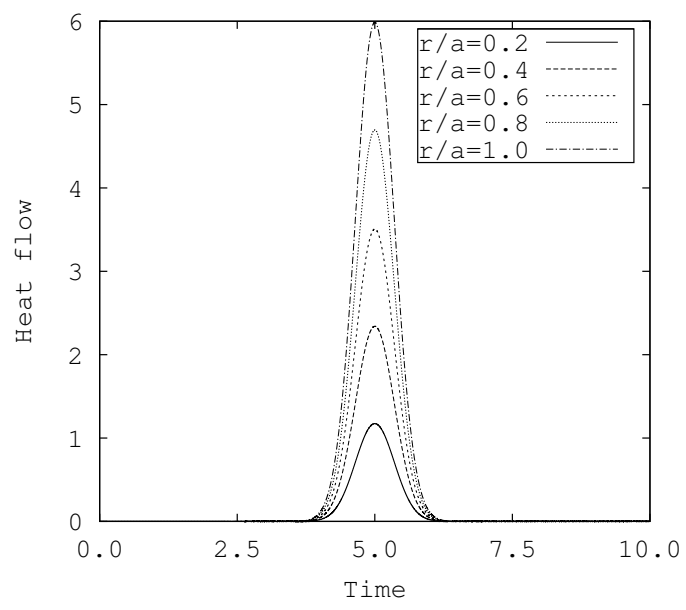

FIG. 2: Evolution of the heat flow $q$ (multiplied by $10^{7}$ ) for the Schwarzschild-like model. The initial conditions are $a(0)=$ $5.0, m(0)=1.0, \omega_{a}(0)=0.0$. The total radiated mass is $M_{r}=10^{-4} m_{a}(0)$.

where $f$ is an arbitrary function of $t$. The expression for $\tilde{p}$ is

$$
\frac{\tilde{p}+\frac{1}{3} \tilde{\rho}}{\tilde{p}+\tilde{\rho}}=\left(1-\frac{8 \pi}{3} \tilde{\rho} r^{2}\right)^{h / 2} k(t),
$$

where $k$ is a function of $t$ to be defined from the boundary condition 12 or $(13)$. Thus, 20 and $(13)$ give

$$
\begin{gathered}
\tilde{\rho}=\frac{3 m_{a}}{4 \pi a^{2}}, \\
\tilde{p}=\frac{\tilde{\rho}}{3}\left\{\frac{\chi_{S}\left(1-2 m_{a} / a\right)^{1 / 2}-3 \psi_{S} \xi}{\psi_{S} \xi-\chi_{S}\left(1-2 m_{a} / a\right)^{1 / 2}}\right\},
\end{gathered}
$$

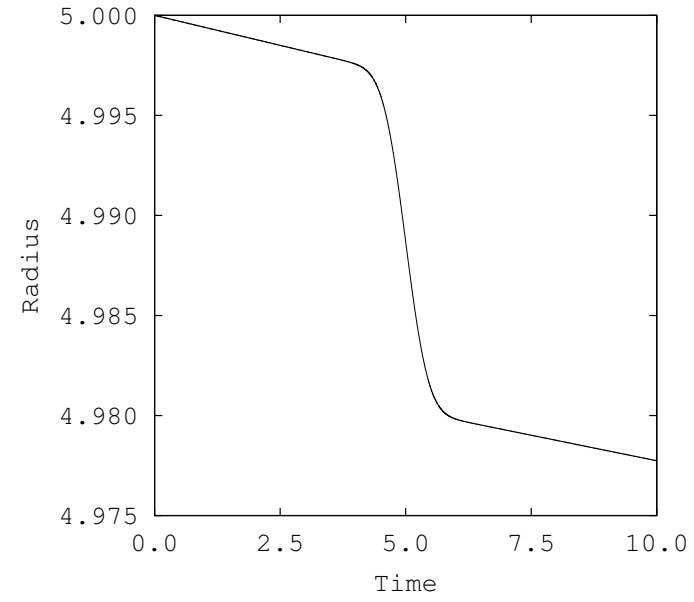

FIG. 3: Evolution of the radius $a$ for the Schwarzschildlike model. The initial conditions are $a(0)=5.0, m(0)=$ 1.0, $\omega_{a}(0)=-0.001$. The total radiated mass is $M_{r}=$ $10^{-2} m_{a}(0)$.

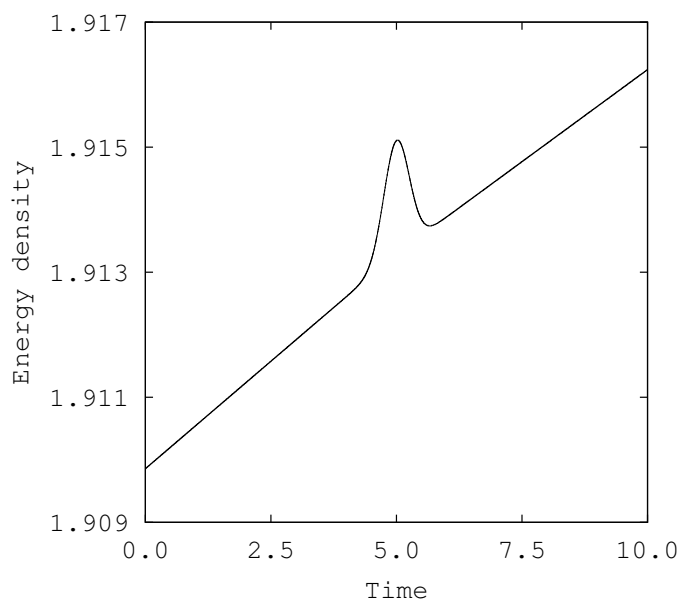

FIG. 4: Evolution of the energy density $\rho$ (multiplied by $10^{3}$ ) for the Schwarzschild-like model. The initial conditions are $a(0)=5.0, m(0)=1.0, \omega_{a}(0)=-0.001$. The total radiated mass is $M_{r}=10^{-2} m_{a}(0)$.

with

$$
\xi=\left[1-\frac{2 m_{a}}{a}\left(\frac{r}{a}\right)^{2}\right]^{1 / 2}
$$

where

$$
\chi_{S}=6\left(\omega_{a}^{2}+1\right) \frac{m_{a}}{a}+8 \pi a^{2} q_{a}\left(1+\omega_{a}\right)^{2},
$$

and

$$
\psi_{S}=2\left(3 \omega_{a}^{2}+1\right) \frac{m_{a}}{a}+8 \pi a^{2} q_{a}\left(1+\omega_{a}\right)^{2} .
$$




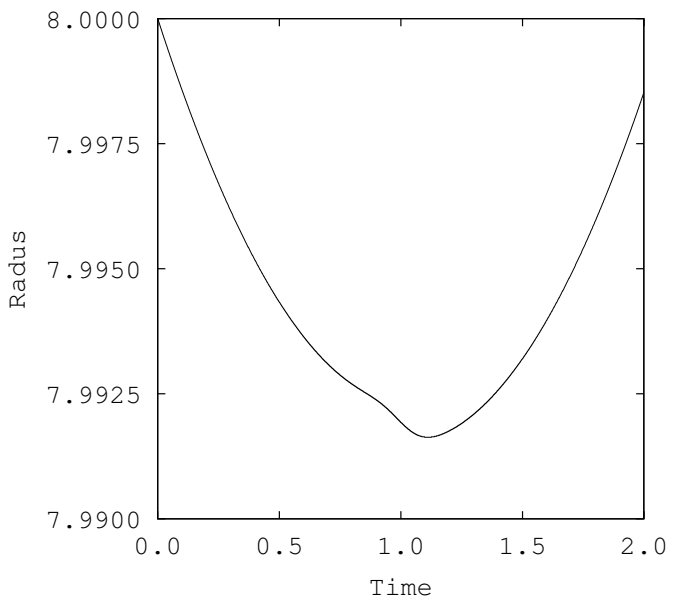

FIG. 5: Evolution of the radius $a$ for the Tolman VI-like model. The initial conditions are $a(0)=8.0, m(0)=1.0$, $\omega_{a}(0)=-0.02$. The total radiated mass is $M_{r}=10^{-4} m_{a}(0)$, with a narrow Gaussian given by $\Sigma=0.01$ with maximum at $t_{0}=1.0$.

Using (17) and (18) it is easy to obtain expressions for $m$ and $\nu$ :

$$
\begin{gathered}
m=m_{a}(r / a)^{3}, \\
e^{\nu}=\left\{\frac{a\left(\chi_{S}\left(1-2 m_{a} / a\right)^{1 / 2}-\psi_{S} \xi\right)}{4 m_{a}}\right\}^{2} .
\end{gathered}
$$

Thus, the system of equations at the surface can be integrated, but it is necessary to specify one function of $t$ and the initial data. We choose

$$
L \equiv 4 \pi a^{2} q_{a}
$$

to be a Gaussian

$$
L=L_{0} e^{-\left(t-t_{0}\right)^{2} / \Sigma^{2}},
$$

with $L_{0}=M_{r} / \sqrt{\Sigma \pi}, t_{0}=5.0$ and $\Sigma=0.25$, which corresponds to a pulse radiating away a fraction of the initial mass $M_{r}$. Therefore, the system can be numerically integrated for the following typical initial conditions:

$$
a(0)=5.0, m_{a}(0)=1.0, \omega_{a}(0)=0.0 .
$$

The integration was done up to some $t$ guaranteeing well behavior of the physical variables, that is, $\rho>0 ; \rho \geq p$; $|\omega|<1 ; \omega, q \in \Re$. Feeding back the numerical values of $a, m_{a}$ and $\omega_{a}$ (and their derivatives) in (17) and (18) we obtain $m$ and $\nu$ (and their partial derivatives) for any value of $r$. Thus, variables $\rho, p, \omega$ and $q$ can be monitored for any piece of the material, via field equations. We calculated them for the values $r / a=0.0,0.2,0.4,0.6,0.8$ and 1.0 .
We explore a complete range of initial conditions and parameters of integration to get physically acceptable results. A radiated mass bigger than $10^{-4}$ and an initially contracting velocity are not permitted. The reason is a complex root calculating the local radial velocity and heat flow for some regions of spacetime. Representative and acceptable results are shown in Figs. 1-2. For this model, the energy density and the radius of the distribution remain almost constant (within six significant figures). These features were not reported in the past using radiation coordinates and lead us to the following model. Our results clearly show that the heat flow keeps the evolution near quasistaticity (slow evolution). Under the same initial compactness used above, that is, $a(0)=5$, we found a possible initial local radial velocity of $\omega_{a}(0)=-10^{-3}$ and a radiated mass of $M_{r}=10^{-2}$, producing now an appreciable change in the energy density and the radius of the distribution. These results are shown in Figs. 3-4. For these conditions only the evolution of a bubble is possible $(r / a \approx 0.99 \rightarrow 1.00)$. We do not observe any evidence of thermal peeling [2], that is, positive velocities (expansion) of outer shells and negative velocities (contraction) of the inner shells. The development of thermal peeling leads to complex roots for the radial velocity.

We consider now other interior seed model based on the Tolman VI interior solution 22. This model corresponds to a highly compressed Fermi gas. Let us take

$$
\begin{gathered}
\tilde{\rho}=\frac{g}{r^{2}}, \\
\tilde{p}=\frac{g[1-9 \alpha(r / a)]}{3[1-\alpha(r / a)] r^{2}},
\end{gathered}
$$

where $g$ and $\alpha$ are functions of $t$, which can be determined using 13). Thus

$$
g=\frac{m_{a}}{4 \pi a}
$$

$$
\begin{gathered}
\alpha=\frac{2 m_{a} / a-3 \beta}{3\left[6 m_{a} / a-\beta\right]} \\
\beta=2 \omega_{a}^{2} \frac{m_{a}}{a}+8 \pi a^{2} q_{a}\left(1+\omega_{a}\right)^{2} .
\end{gathered}
$$

Once the metric functions are obtained from (17) and (18), the system of equations at the surface can be again numerically integrated for the following initial conditions:

$$
a(0)=8.0, m_{a}(0)=1.0, \omega_{a}(0)=-0.02 .
$$

As before, a radiated mass bigger than $10^{-4}$ is not permitted. But even more, now it is not possible to go inside the distribution without violating real values assumption from the beginning. For that reason the Gaussian has been set to $\Sigma=0.01$ and $t_{0}=1$. At the surface, see Fig. 5 , the results are as expected. 


\section{CONCLUSIONS}

In this paper we considered heat flow as a transport mechanism in the PQSA. Heat flow produces a stable configuration, which is the opposite effect of viscosity 4]. This result indicates that a combination of viscosity (anisotropy) with heat flow may be crucial for gravitational collapse or at least just out of equilibrium, where we expect the PQSA is a good approach. We did additional tests including anisotropy but its effect is marginal. For distributions far from equilibrium we find that heat flow is a very restrictive transport mechanism.

These results are apparently different for the same configurations in radiation coordinates [16]-[19]. If the initial distribution is in equilibrium the transition from static to postquasistatic, in radiation coordinates, al- lows the sphere to "instantaneously" bypass diffusion stressing. But in Schwarzschild coordinates we can follow the transition from the static configuration to the postquasistatic with more resolution. Diffunding radiation strongly interacts with matter. As a result, the interior of the distribution is not permitted to go far from equilibrium. When including heat flow, there is not PQSA except very close to or at the surface.

\section{Acknowledgments}

C. P. acknowledges the computing resources provided by the Victorian Partnership for Advanced Computation (VPAC).
[1] D. Kazanas and D. Schramm Sources of gravitational Radiation (Cambridge: Cambridge University Press, 1979).

[2] L. Herrera and A. Di Prisco, Phys. Rev. D, 55, 2044 (1997).

[3] J. Lattimer, Nucl. Phys. A 478, 199 (1988).

[4] C. Peralta, L. Rosales, B. Rodríguez and W. Barreto, Phys. Rev. D, 81, 104021 (2010).

[5] W. Barreto, B. Rodríguez and H. Martínez, Ap. Sp. Sc., 282, 581 (2002).

[6] L. Herrera, W. Barreto, A. Di Prisco and N. Santos, Phys. Rev. D, 65104004 (2002).

[7] W. Barreto Phys. Rev. D 79, 10, 107502 (2009).

[8] W. Barreto, L. Castillo and E. Barrios Phys. Rev. D 80, 084007 (2009).

[9] W. Barreto, L. Castillo and E. Barrios, Bondian frames to couple matter with radiation, published on line in General Relativity and Gravitation, 06 March (2010).

[10] L. Herrera, J. Jiménez and G. Ruggeri, Phys. Rev. D, 22, 2305 (1980).

[11] L. Herrera and L. Núñez, Fund. Cosmic Phys., 14, 235 (1990).
[12] W. Barreto, L. Herrera, and L. Núñez, Ap. J. 375, 663 (1991).

[13] W. Barreto, L. Herrera, and N. Santos, Ap. Sp. Sc. 187, 271 (1992).

[14] L. Herrera, A. Melfo, L. A. Núñez, and A. Patiño, Ap. J. 421, 677 (1994).

[15] W. Barreto and A. Da Silva, Gen. Rel. Grav. 28, 735 (1996).

[16] L. Herrera, J. Jiménez and M. Esculpi, Phys. Rev. D 36, 2986 (1987).

[17] W. Barreto, L. Herrera and N. Santos. Ap. J. 344, 158 (1989).

[18] W. Barreto, L. Herrera and N. Santos, Ap. Sp. Sc. 187, 271 (1992).

[19] W. Barreto, C. Peralta and L. Rosales, Phys. Rev. D 59, 024008 (2000).

[20] J. Martínez, Phys. Rev. D, 53, 6921 (1996).

[21] H. Bondi, Proc. Royal Soc. London, A281, 39 (1964).

[22] R. C. Tolman, Phys. Rev., 55, 364 (1939). 